\title{
The effect of a quantitative resuscitation strategy on mortality in patients with sepsis: A meta-analysis*
}

\author{
Alan E. Jones, MD, Michael D. Brown, MD, MSc, Stephen Trzeciak, MD, MPH, Nathan I. \\ Shapiro, MD, MPH, John S. Garrett, MD, Alan C. Heffner, MD, and Jeffrey A. Kline, MD [on \\ behalf of on behalf of the Emergency Medicine Shock Research Network investigators] \\ From the Department of Emergency Medicine (AEJ, JSG, ACH, JAK), Carolinas Medical Center, \\ Charlotte, NC; Grand Rapids MERC/Michigan State University Program in Emergency Medicine \\ (MDB), Grand Rapids, MI; Department of Emergency Medicine (NIS), Beth Israel Deaconess \\ Medical Center, Boston, MA; and Departments of Emergency Medicine and Medicine, Division of \\ Critical Care Medicine (ST), UMDNJ-Robert Wood Johnson Medical School at Camden, Cooper \\ University Hospital, Camden, NJ.
}

\section{Abstract \\ Objective-Quantitative resuscitation consists of structured cardiovascular intervention targeting predefined hemodynamic end points. We sought to measure the treatment effect of quantitative resuscitation on mortality from sepsis.}

Data Sources-We conducted a systematic review of the Cochrane Library, MEDLINE, EMBASE, CINAHL, conference proceedings, clinical practice guidelines, and other sources using a comprehensive strategy.

Study Selection-We identified randomized control trials comparing quantitative resuscitation with standard resuscitation in adult patients who were diagnosed with sepsis using standard criteria. The primary outcome variable was mortality.

Data Abstraction-Three authors independently extracted data and assessed study quality using standardized instruments; consensus was reached by conference. Preplanned subgroup analysis required studies to be categorized based on early (at the time of diagnosis) vs. late resuscitation implementation. We used the chi-square test and $I^{2}$ to assess for statistical heterogeneity $(p<0.10$, $\left.I^{2}>25 \%\right)$. The primary analysis was based on the random effects model to produce pooled odds ratios with $95 \%$ confidence intervals.

Results-The search yielded 29 potential publications; nine studies were included in the final analysis, providing a sample of 1001 patients. The combined results demonstrate a decrease in mortality (odds ratio $0.64,95 \%$ confidence interval $0.43-0.96$ ); however, there was statistically significant heterogeneity $\left(p=0.07, I^{2}=45 \%\right)$. Among the early quantitative resuscitation studies (n $=6)$ there was minimal heterogeneity $\left(p=0.40, I^{2}=2.4 \%\right)$ and a significant decrease in mortality (odds ratio $0.50,95 \%$ confidence interval $0.37-0.69$ ). The late quantitative resuscitation studies (n $=3$ ) demonstrated no significant effect on mortality (odds ratio 1.16 , 95\% confidence interval 0.60 2.22).

Conclusion-This meta-analysis found that applying an early quantitative resuscitation strategy to patients with sepsis imparts a significant reduction in mortality.

\footnotetext{
*See also p. 2932.

Copyright $\odot 2008$ by the Society of Critical Care Medicine and Lippincott Williams \& Wilkins

For information regarding this article, E-mail: alan.jones@carolinas.org
} 


\section{Keywords}

sepsis; septic shock; mortality; resuscitation; meta-analysis

Quantitative resuscitation in critically ill patients consists of structured cardiovascular intervention with intravascular volume expansion and vasoactive agent support to achieve explicit predefined physiologic or supraphysiologic end points. The concept of quantitative resuscitation (also referred to as hemodynamic optimization, goal-oriented resuscitation, or goal-directed therapy) as a treatment strategy to improve clinical outcome was first reported by Shoemaker et al. (1) in high risk surgery patients. Since this sentinel report, multiple other investigators have published randomized clinical trials investigating the effect of quantitative resuscitation in various critically ill patient populations, including those with sepsis.

In the United States, 750,000 people experience severe sepsis each year, of whom 500,000 require intensive or intermediate level care, and 30\% die (2). During the last 30 years numerous new therapeutic strategies for severe sepsis have been investigated. Unfortunately, few therapies have demonstrated significant reduction in mortality in patients with severe sepsis (3). However, a randomized control trial of 263 patients by Rivers et al. (4) reported a $16 \%$ absolute reduction in in-hospital mortality among severe sepsis and septic shock patients who received early quantitative resuscitation when compared with standard resuscitation. As a result of this trial, the Surviving Sepsis Campaign guidelines recommend that a quantitative resuscitation strategy should be implemented at the time of recognition of severe sepsis (5). Despite its endorsement by 11 professional societies, the recommendation has produced significant controversy, in part because it relied heavily on one study (6-10).

Scientific knowledge is cumulative; therefore new research findings should be interpreted based on what is already known (11). Recognizing that clinical trials of quantitative resuscitation were published for nearly two decades before the Rivers et al. study, we hypothesized that their results could be aggregated to produce more definitive conclusions about the treatment effect of quantitative resuscitation in sepsis. Therefore, we conducted a meta-analysis to examine the available evidence investigating a quantitative resuscitation strategy for patients with sepsis, to determine the treatment effect of such a strategy on mortality, and to determine whether the timing of implementation of quantitative resuscitation impacts mortality.

\section{METHODS}

\section{Search Strategy for Identification of Studies}

A written protocol following recommended guidelines that was finalized before beginning the study was followed (12). We searched the Cochrane Library, MEDLINE (1965-August 2007), EMBASE (1974-August 2007), and CINHAL (1982-August 2007) using the search terms (sepsis or septic shock or severe sepsis or critical care) and (hemodynamics or resuscitation or resuscitation end points or oxygen delivery or goal-oriented therapy or goal-directed resuscitation or early goal-directed therapy) and clinical trial. The search was updated in April 2008. To identify potential unpublished data we contacted two experts in the field of sepsis resuscitation, we hand searched abstracts from the Society of Critical Care Medicine, American College of Chest Physicians, American College of Emergency Physicians and Society for Academic Emergency Medicine annual meetings from 1997 to 2007, we reviewed published practice guidelines for sepsis care from 2000 to 2007, and searched websites containing details for clinical trial registration. Additionally, reference lists of the articles chosen for inclusion and the reference lists of previous reviews (13-18) were screened to identify further studies for inclusion. 


\section{Inclusion Criteria}

We considered studies eligible for review regardless of language or publication type, if they were randomized control trials of adults (age $>17 \mathrm{yr}$ ) with a presumptive or confirmed diagnosis of sepsis who were treated in an experimental study using: 1) a clearly defined intervention consisting of a structured cardiovascular resuscitation protocol administered to achieve predefined hemodynamic end points; and 2) a control group in which subjects received standard of care therapy. We included subpopulations of sepsis patients from studies with large heterogeneous populations, if the subpopulations met our inclusion criteria. Reviews, correspondence, editorials, and nonhuman studies were excluded; however, their reference lists were screened to identify further studies for inclusion. We attempted to contact corresponding authors for clarification of data extraction or quality assessment if not clear from the published article or abstract.

\section{Study Selection and Data Abstraction}

Two independent reviewers (A.E.J. and J.S.G.) screened the titles and abstracts of identified studies for potential eligibility (19). After the relevance screen the two reviewers compared their exclusion logs to determine whether there was disagreement; the Kappa statistic was used to assess interobserver agreement. In cases of disagreement, a third reviewer assessed the abstract and a consensus was reached by conference between the three reviewers. All studies deemed potentially relevant were obtained and the full manuscripts were reviewed for inclusion. Three reviewers (J.A.K., N.I.S., S.T.) independently abstracted data on all patient populations, interventions, and outcomes using a standardized data collection form. Any disagreements in these processes were resolved by consensus.

\section{Assessment of Quality}

Study quality was assessed using two major categories:

1. Appropriate patient selection by identification of sepsis using accepted diagnostic criteria (20): grade A—used standard criteria, grade B—used nonstandard criteria, grade $\mathrm{C}$ - unknown.

2. Internal validity assessment based on concealment of allocation: grade $\mathrm{A}$-adequate, grade $\mathrm{B}$ - unclear, grade $\mathrm{C}$-inadequate. The Cochrane Handbook (12) provides definitions for each category as follows: grade A-allocation concealment was adequately reported (centralized randomization either by a central office; sequential administration of coded containers to enrolled participants; on-site computer; serially numbered sealed opaque envelopes); grade B-allocation concealment is not described, but it is mentioned in the text that the study is randomized; grade $\mathrm{C}-$ allocation concealment was inadequate (alternation; dates of birth; days of the week; any allocation that is entirely transparent before allocation).

Blinding was not considered essential because it was recognized that masking clinicians and patients from a complex intervention such as a quantitative resuscitation protocol would be extremely difficult. In addition, the potential for measurement bias was minimal given that the primary outcome was death.

\section{Main Outcome Measure}

We defined mortality at the end of the time frame reported by the authors to be the primary dependent variable for analysis. In that event, authors reported mortality at more than one time point we used in-hospital mortality preferentially. 


\section{Primary Statistical Analysis}

All data were entered into Review Manager Software (version 4.2. Copenhagen: The Nordic Cochrane Centre, The Cochrane Collaboration, 2003). Heterogeneity was tested using a chisquare test with a $p$ value $<0.10$ to indicate significant heterogeneity between the trials. In addition, $I^{2}$ was calculated to provide an estimate of the variability across studies beyond that due to chance (21). The results of studies were pooled using a random effects model. The individual and pooled statistics were calculated as odds ratios (OR) with $95 \%$ confidence intervals (CI). We constructed a funnel plot and performed Egger regression (22) to detect the presence of publication bias. However, the authors acknowledge that statistical tests used to identify publication bias have low power and any asymmetry identified in a funnel plot may be the result of numerous factors other than publication bias $(23,24)$.

\section{Subgroup and Sensitivity Analyses}

We hypothesized that studies implementing early quantitative resuscitation, defined as therapy implemented at the time of recognition of sepsis or within $24 \mathrm{hr}$, may have different results than studies that reported late quantitative resuscitation (initiated after $24 \mathrm{hr}$ or unknown/ unreported timing). Considering this obvious clinical heterogeneity, subgroup analysis was planned a priori for these two groups of studies based on timing to quantitative resuscitation intervention (early vs. late). We also planned sensitivity analysis to determine whether including only studies with high quality of conduct or reporting yielded different results. Studies reporting adequate concealment of allocation (grade A) were considered "high quality." In addition, we performed a post hoc (not described in our original protocol) sensitivity analysis in which we excluded each study individually and reanalyzed the pooled OR among the early subgroup to determine whether any individual study may be influencing the results. Finally, a sensitivity analysis was performed to examine the use of a fixed effect vs. random effects model.

\section{RESULTS}

\section{Search and Selectio}

The comprehensive search yielded a total of 903 relevant publications; details of the search and study selection are shown in Figure 1 and Table 1.

\section{Inclusion}

After the relevance search (blinded interobserver agreement $98.7 \%$ with 12 disagreements of 903 articles, $\kappa=0.7$ ) a complete manuscript review was performed on the remaining 29 articles. Nine studies were included in the final analysis providing a total sample of 1001 subjects $(4$, 25-32). Two full length articles in Chinese were translated to English, prior to analysis (32, $33)$.

\section{Study Descriptions}

The incidence of death among patients in the quantitative resuscitation groups ranged from $13 \%$ to $74 \%$ with a mean of $47 \%$ and in the control groups ranged from $27 \%$ to $72 \%$ with a mean of $57 \%$. Eight studies took place in the intensive care unit and one in the emergency department (ED). Detailed description of included studies is shown in Table 2.

\section{Quality Assessment}

Three independent observers (JAK, NIS, ST) graded the studies with respect to the key quality variable of concealment of allocation; consensus was reached by conference. Four studies were assigned grade $\mathrm{A}$, three studies were assigned grade $\mathrm{B}$ and two were assigned grade $\mathrm{C}$ (Table 2). 


\section{Analyses}

When all nine studies were considered, there was evidence of both statistical heterogeneity $\left(p=0.07, I^{2}=45 \%\right)$ and clinical heterogeneity among the studies. The summary OR demonstrates a significant reduction in mortality (OR 0.64, 95\% CI $0.43-0.96)$ among subjects receiving quantitative resuscitation (Fig. 2). To assess for potential publication bias, a funnel plot of all included studies is provided in Figure 3. Egger regression analysis revealed no significant indication of publication bias (intercept $=0.61,95 \% \mathrm{CI}-2.4-3.6, p=0.65$ ).

Studies within the two subgroups defined a priori (early vs. late) were combined and the results are shown in Figure 2. Among the early quantitative resuscitation studies ( $\mathrm{n}=6$ studies), there was minimal heterogeneity $\left(p=0.40, I^{2}=2.4 \%\right)$. The overall mortality rate in the early quantitative resuscitation group was $39 \%$. Patients randomized to receive early quantitative resuscitation compared with standard resuscitation had a significantly lower mortality rate (OR $0.50,95 \%$ CI $0.37-0.69$ ). Results were robust to sensitivity analysis; when the lower quality studies (grades B and C) were excluded, the results in the early quantitative resuscitation subgroup were similar (OR 0.60, 95\% CI 0.35-1.0). In the late quantitative resuscitation group $(\mathrm{n}=3$ studies) the overall mortality rate was $64 \%$. Patients randomized to late quantitative resuscitation did not have a significantly different mortality rate compared with standard resuscitation (OR 1.16, 95\% CI $0.60-2.22$ ). None of the above point estimates were significantly different when using a fixed effect model.

Given that early quantitative resuscitation had such a clear impact on mortality, we performed a post hoc sensitivity analysis in which we step wise excluded each study individually and reanalyzed the pooled OR for the early subgroup to determine whether any individual study had an overwhelming influence on the results. None of the point estimates changed during this analysis and the $95 \%$ CI only widened slightly but remained well below 1.0.

\section{DISCUSSION}

This meta-analysis evaluates the treatment effect of using a quantitative resuscitation strategy in the treatment of patients with sepsis. Using pooled data from nine studies that randomized a total of 1001 subjects, we found the magnitude of the decrease in mortality (OR 0.50 with the upper limit 95\% CI 0.69) was profound when the resuscitation strategy was implemented early. The benefits of a quantitative resuscitation strategy seem to be completely lost if the intervention is initiated late. Our results provide the strongest support to date for the Surviving Sepsis Campaign recommendation of providing quantitative resuscitation at the time of severe sepsis recognition (5).

This systematic review used both a comprehensive search and standard methods for summarizing the treatment effect of a quantitative resuscitation strategy in patients with sepsis. Our subgroup analysis of early vs. late quantitative resuscitation pooled studies based on the reported timing of the resuscitation intervention and this timing may not have been a part of the original study methods. The limitations of making inferences based on between-study rather than within-study comparisons have been well-described but our subgroup analysis was limited to one hypothesis and was developed a priori based on the sound biological plausibility that a quantitative resuscitation strategy may require early initiation to be effective (34).

The importance of the treatment benefit reported in this meta-analysis is underscored by the continued high mortality rate among patients with severe sepsis. Three recently published randomized control trials of medication treatments for severe sepsis and septic shock reported 28 day mortality rates between $30 \%$ and $60 \%$ (35-37). Furthermore, it is estimated that severe sepsis cases account for $>500,000$ ED visits annually and that these patients stay an average of $5 \mathrm{hr}$ in the $\mathrm{ED}$ (38). Our findings suggest that applying an early quantitative resuscitation 
strategy in these patients at the time of recognition, such as in the ED or after immediate transfer to an intensive care unit, would impart a substantial survival benefit.

Any meta-analysis is prone to bias due to its retrospective approach; however, we used a number of important steps in an attempt to minimize its impact. First, we followed a protocol that was written before starting the search and analysis. Second, we used independent observers to both abstract data and grade study quality. We should note that in our original protocol we proposed to grade the quality of the studies based on the criteria proposed by Jadad et al. (39). However, these criteria were intended to be applied to classic therapeutic trials, such as medication treatment studies, and after attempts to apply these criteria to the trials reviewed in this meta-analysis, the consensus of the investigators was that they did not accurately represent the quality of the studies. Therefore we report the Jadad score but did not use it as our primary quality measure. Third, we constructed a funnel plot to assess for publication bias. Visual inspection of the plot reveals a lack of studies in the left lower quadrant indicating the potential that smaller studies demonstrating a significant reduction in mortality were not identified. Finally, our meta-analysis addressed all of the suggested necessary components of the AMSTAR methodologic quality measurement tool for systematic reviews (40). A limitation of this meta-analysis is the possibility that unreported cointerventions (e.g., corticosteroids or intensive insulin therapy) may have influenced the results of included studies; there was no practical way to capture or control for such interventions in this meta-analysis.

Several previous systematic reviews have addressed the treatment effect of quantitative resuscitation or hemodynamic optimization in critically ill patients $(13-14,16-17)$. Two of these studies investigated the effect of attaining either physiologic or supraphysiologic values in broad groups of critically ill patients such as pre- and postsurgical, trauma, sepsis, and acute respiratory distress syndrome patients $(14,16-17)$. Only one of the reviews specifically investigated sepsis, however, the objective of this review was to determine the variation in the resuscitation goals targeted and whether this variation was associated with differences in outcome (13). Thus the present meta-analysis is markedly different from these prior systematic reviews in that we investigated the treatment effect of a quantitative resuscitation strategy only in patients with sepsis and analyzed the studies with respect to temporal timing of the intervention.

It is important to note that several barriers to adoption of a quantitative resuscitation strategy for sepsis in the ED have been identified $(4,41,42)$. In fact, the majority of the studies in the early subgroup of this meta-analysis did not start quantitative resuscitation until intensive care unit admission leaving the possibility of some delay in treatment initiation among these studies. Although implementation requires that organizational and resource barriers be overcome, a number of reports have documented successful implementation of such programs (43-46). Additionally, we observed distinct variations in the goals or end points targeted among the studies included in this analysis (Table 2). It was not an aim of the present investigation to determine whether any particular resuscitation end point was superior but rather to determine whether a quantitative resuscitation strategy (regardless of the endpoint) affected survival. Future investigations should compare the relative efficacy of various quantitative resuscitation endpoints in a randomized study design.

\section{CONCLUSION}

Data synthesized from nine randomized controlled trials demonstrate a clear survival benefit afforded by quantitative resuscitation to treat sepsis at or near the time of recognition. The benefit of quantitative resuscitation seems to be completely lost if the intervention is initiated late. Future studies should investigate the optimal endpoints of quantitative resuscitation for sepsis. 


\section{ACKNOWLEDGMENTS}

We thank the following corresponding authors: Luciano Gattinoni, Mihae Yu, and Rodney Durham. In addition, we thank Ming Qi, MD for help with article translation and Bridget Loven for help with the literature search.

Supported, in part, by a grant from the National Institute of General Medical Sciences K23GM76652 (AEJ).

Dr. Jones has received unrestricted research support from Critical Biologies Corporation. Dr. Shapiro has received consulting fees, honoraria, and grants from Eli Lilly. Dr. Kline is inventor on US Patent 7,083,574. The remaining authors have not disclosed any potential conflicts of interest.

\section{REFERENCES}

1. Shoemaker WC, Appel PL, Kram HB, et al. Prospective trial of supranormal values of survivors as therapeutic goals in high-risk surgical patients. Chest 1988;94:1176-1186. [PubMed: 3191758]

2. Angus DC, Linde-Zwirble WT, Lidicker J, et al. Epidemiology of severe sepsis in the United States: Analysis of incidence, outcome, and associated costs of care. Crit Care Med 2001;29:1303-1310. [PubMed: 11445675]

3. Centers for Disease Control and Prevention. Current trends increase in national hospital discharge survey rates for septicemia-United States, 1979-1987. MMWR 1991;39:31-34.

4. Rivers E, Nguyen B, Havstad S, et al. Early goal-directed therapy in the treatment of severe sepsis and septic shock. N Engl J Med 2001;345:1368-1677. [PubMed: 11794169]

5. Dellinger RP, Levy MM, Carlet JM, et al. Surviving sepsis campaign: International guidelines for management of severe sepsis and septic shock: 2008. Crit Care Med 2008;36:296-327. [PubMed: 18158437]

6. Peake S, Webb S, Delaney A. Early goal-directed therapy of septic shock: We honestly remain skeptical. Crit Care Med 2007;35:994-995. [PubMed: 17421118]

7. Marik PE, Varon J. Goal-directed therapy for severe sepsis. N Engl J Med 2002;346:1025-1026. [PubMed: 11919315]

8. Ho BC, Bellomo R, McGain F, et al. The incidence and outcome of septic shock patients in the absence of early-goal directed therapy. Crit Care 2006;10:R80. [PubMed: 16704743]

9. Abroug F, Besbes L, Nouira S. Goal-directed therapy for severe sepsis. N Engl J Med 2002;346:10251026. [PubMed: 11924658]

10. Sarkar S, Kupfer Y, Tessler S. Goal-directed therapy for severe sepsis. N Engl J Med 2002;346:10251026. [PubMed: 11924659]

11. Clarke M. Doing new research? Don't forget the old. PLoS Med 2004;1:e35. [PubMed: 15578106]

12. Higgins, JPT.; Green, S., editors. Cochrane handbook for systematic reviews of interventions 4.2.6 [Updated September 2006]. Chichester, UK, Wiley: Cochrane Library; 2006.

13. Sevransky JE, Nour S, Susia GM, et al. Hemodynamic goals in randomized clinical trials in patients with sepsis: A systematic review of the literature. Crit Care 2007;11:R67. [PubMed: 17584921]

14. Poeze M, Greve JW, Ramsay G. Meta-analysis of hemodynamic optimization: Relationship to methodological quality. Crit Care 2005;9:R771-R779. [PubMed: 16356226]

15. Macias WL, Nelson DR, Williams M, et al. Lack of evidence for qualitative treatment by disease severity interactions in clinical studies of severe sepsis. Crit Care 2005;9:R607-R622. [PubMed: 16280057]

16. Kern JW, Shoemaker WC. Meta-analysis of hemodynamic optimization in high-risk patients. Crit Care Med 2002;30:1686-1692. [PubMed: 12163777]

17. Heyland DK, Cook DJ, King D, et al. Maximizing oxygen delivery in critically ill patients: A methodologic appraisal of the evidence. Crit Care Med 1996;24:517-524. [PubMed: 8625644]

18. Otero RM, Nguyen HB, Huang DT, et al. Early goal-directed therapy in severe sepsis and septic shock revisited: Concepts, controversies, and contemporary findings. Chest 2006;130:1579-1595. [PubMed: 17099041]

19. Meade MO, Richardson WS. Selecting and appraising studies for a systematic review. Ann Intern Med 1997;127:531-537. [PubMed: 9313021] 
20. Bone RC, Balk RA, Cerra FB, et al. Definitions for sepsis and organ failure and guidelines for the use of innovative therapies in sepsis. The ACCP/SCCM Consensus Conference Committee. American College of Chest Physicians/Society of Critical Care Medicine. Chest 1992;101:16441655. [PubMed: 1303622]

21. Higgins JP, Thompson SG, Deeks JJ, et al. Measuring inconsistency in meta-analyses. BMJ 2003;327:557-560. [PubMed: 12958120]

22. Egger M, Smith GD, Schneider M, et al. Bias in meta-analysis detected by a simple, graphical test. BMJ 1997;315:629-634. [PubMed: 9310563]

23. Terrin N, Schmid CH, Lau J. In an empirical evaluation of the funnel plot, researchers could not visually identify publication bias. J Clin Epidemiol 2005;58:894-901. [PubMed: 16085192]

24. Tang JL, Liu JL. Misleading funnel plot for detection of bias in meta-analysis. J Clin Epidemiol 2000;53:477-484. [PubMed: 10812319]

25. Lin SM, Huang CD, Lin HC, et al. A modified goal-directed protocol improves clinical outcomes in intensive care unit patients with septic shock: A randomized controlled trial. Shock 2006;26:551557. [PubMed: 17117128]

26. Alia I, Esteban A, Gordo F, et al. A randomized and controlled trial of the effect of treatment aimed at maximizing oxygen delivery in patients with severe sepsis or septic shock. Chest 1999;115:453461. [PubMed: 10027447]

27. Yu M, Levy MM, Smith P, et al. Effect of maximizing oxygen delivery on morbidity and mortality rates in critically ill patients: A prospective, randomized, controlled study. Crit Care Med 1993;21:830-838. [PubMed: 8504649]

28. Yu M, Burchell S, Hasaniya NW, et al. Relationship of mortality to increasing oxygen delivery in patients $>$ or $=50$ years of age: A prospective, randomized trial. Crit Care Med 1998;26:1011-1019. [PubMed: 9635648]

29. Tuchschmidt J, Fried J, Astiz M, et al. Elevation of cardiac output and oxygen delivery improves outcome in septic shock. Chest 1992;102:216-220. [PubMed: 1623756]

30. Gattinoni L, Brazzi L, Pelosi P, et al. A trial of goal-oriented hemodynamic therapy in critically ill patients. $\mathrm{SvO}_{2}$ Collaborative Group. N Engl J Med 1995;333:1025-1032. [PubMed: 7675044]

31. Hayes MA, Timmins AC, Yau EH, et al. Elevation of systemic oxygen delivery in the treatment of critically ill patients. N Engl J Med 1994;330:1717-1722. [PubMed: 7993413]

32. Xiao-Zhi W, Chang-Jun L, Fu-Quan GAO. Efficacy of goal-directed therapy in the treatment of septic shock. Chin Crit Care Med 2007;18:661-664.

33. Zheng-Yu HE, Yuan GAO, Xiang-Rui W, et al. Clinical evaluation of early goal directed therapy in septic shock. Chin Crit Care Med 2007;19:14-16.

34. Oxman AD, Guyatt GH. A consumer's guide to subgroup analyses. Ann Intern Med 1992;116:7884. [PubMed: 1530753]

35. Bernard GR, Vincent JL, Laterre PF, et al. Efficacy and safety of recombinant human activated protein C for severe sepsis. N Engl J Med 2001;344:699-709. [PubMed: 11236773]

36. Annane D, Sebille V, Charpentier C, et al. Effect of treatment with low doses of hydrocortisone and fludrocortisone on mortality in patients with septic shock. JAMA 2002;288:862-871. [PubMed: 12186604]

37. Annane D, Vignon P, Renault A, et al. Norepinephrine plus dobutamine versus epinephrine alone for management of septic shock: A randomised trial. Lancet 2007;370:676-684. [PubMed: 17720019]

38. Wang HE, Shapiro NI, Angus DC, et al. National estimates of severe sepsis in United States emergency departments. Crit Care Med 2007;35:1928-1936. [PubMed: 17581480]

39. Jadad AR, Moore RA, Carroll D, et al. Assessing the quality of reports of randomized clinical trials: Is blinding necessary? Control Clin Trials 1996;17:1-12. [PubMed: 8721797]

40. Shea BJ, Grimshaw JM, Wells GA, et al. Development of AMSTAR: A measurement tool to assess the methodological quality of systematic reviews. BMC Med Res Methodol 2007;7:10. [PubMed: 17302989]

41. Jones AE, Kline JA. Use of goal-directed therapy for severe sepsis and septic shock in academic emergency departments. Crit Care Med 2005;33:1888-1889. [PubMed: 16096485] 
42. Carlbom DJ, Rubenfeld GD. Barriers to implementing protocol-based sepsis resuscitation in the emergency department-Results of a national survey. Crit Care Med 2007;35:2525-2535. [PubMed: 18075366]

43. Jones AE, Focht A, Horton JM, et al. Prospective external validation of the clinical effectiveness of an emergency department-based early goal directed therapy protocol for severe sepsis and septic shock. Chest 2007;132:425-432. [PubMed: 17573521]

44. Trzeciak S, Dellinger RP, Abata NL, et al. Translating research to clinical practice: A 1-year experience with implementing early goal-directed therapy for septic shock in the emergency department. Chest 2006;129:225-235. [PubMed: 16478835]

45. Micek ST, Roubinian N, Heuring T, et al. Before-after study of a standardized hospital order set for the management of septic shock. Crit Care Med 2006;34:2707-2713. [PubMed: 16943733]

46. Shapiro NI, Howell MD, Talmor D, et al. Implementation and outcomes of the Multiple Urgent Sepsis Therapies (MUST) protocol. Crit Care Med 2006;34:1025-1032. [PubMed: 16484890]

\section{Appendix}

\section{APPENDIX}

The Emergency Medicine Shock Research Network investigators are Alan E. Jones, Stephen Trzeciak, Nathan I. Shapiro, and Jeffrey A. Kline. 


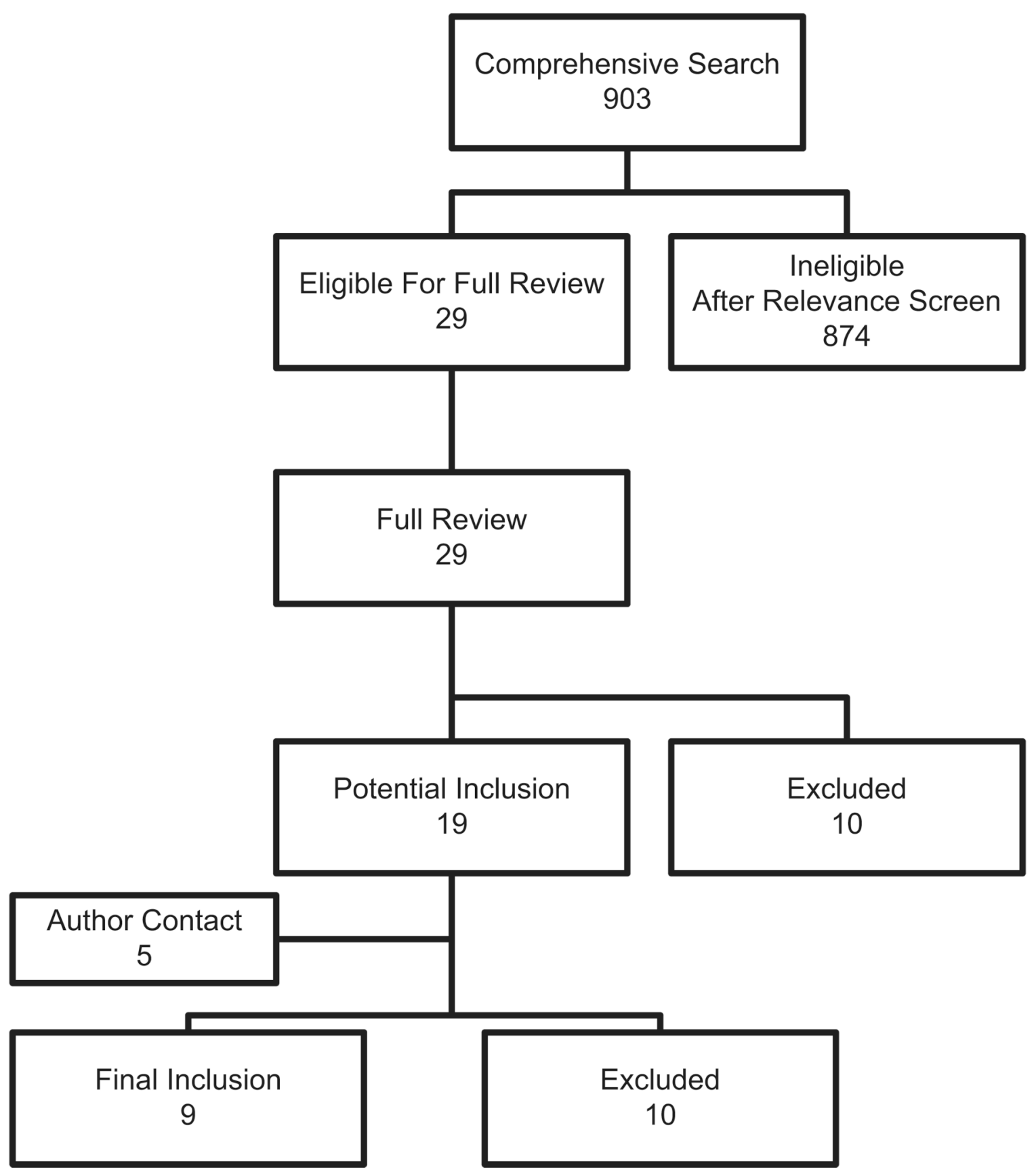

Figure 1.

Search, inclusion, and exclusion flow diagram. 
Review: Quantitative Resuscitation Strategy for Sepsis

Comparison: 01 Quantitative Resuscitation vs. Standard Care

Outcome: 01 Mortality

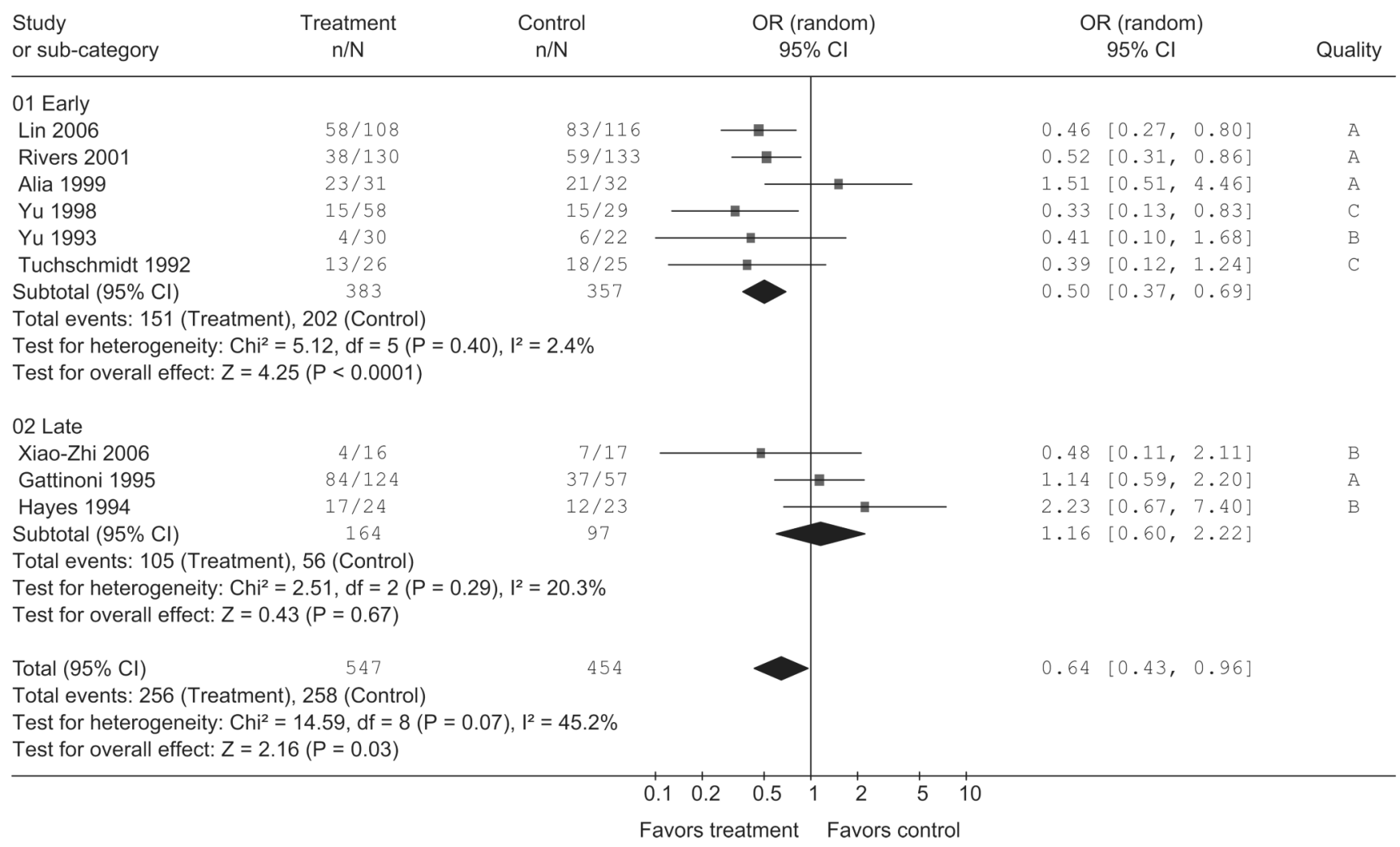

Figure 2.

Forest plot of all included studies and of the subgroup analysis of early vs. late quantitative resuscitation using a random effects model. $O R$, odds ratio; $C I$, confidence interval; $d f$, degrees of freedom. 
Review: Quantitative Resuscitation Strategy for Sepsis

Comparison: 01 Quantitative Resuscitation vs. Standard Care

Outcome: 04 Funnel Plot

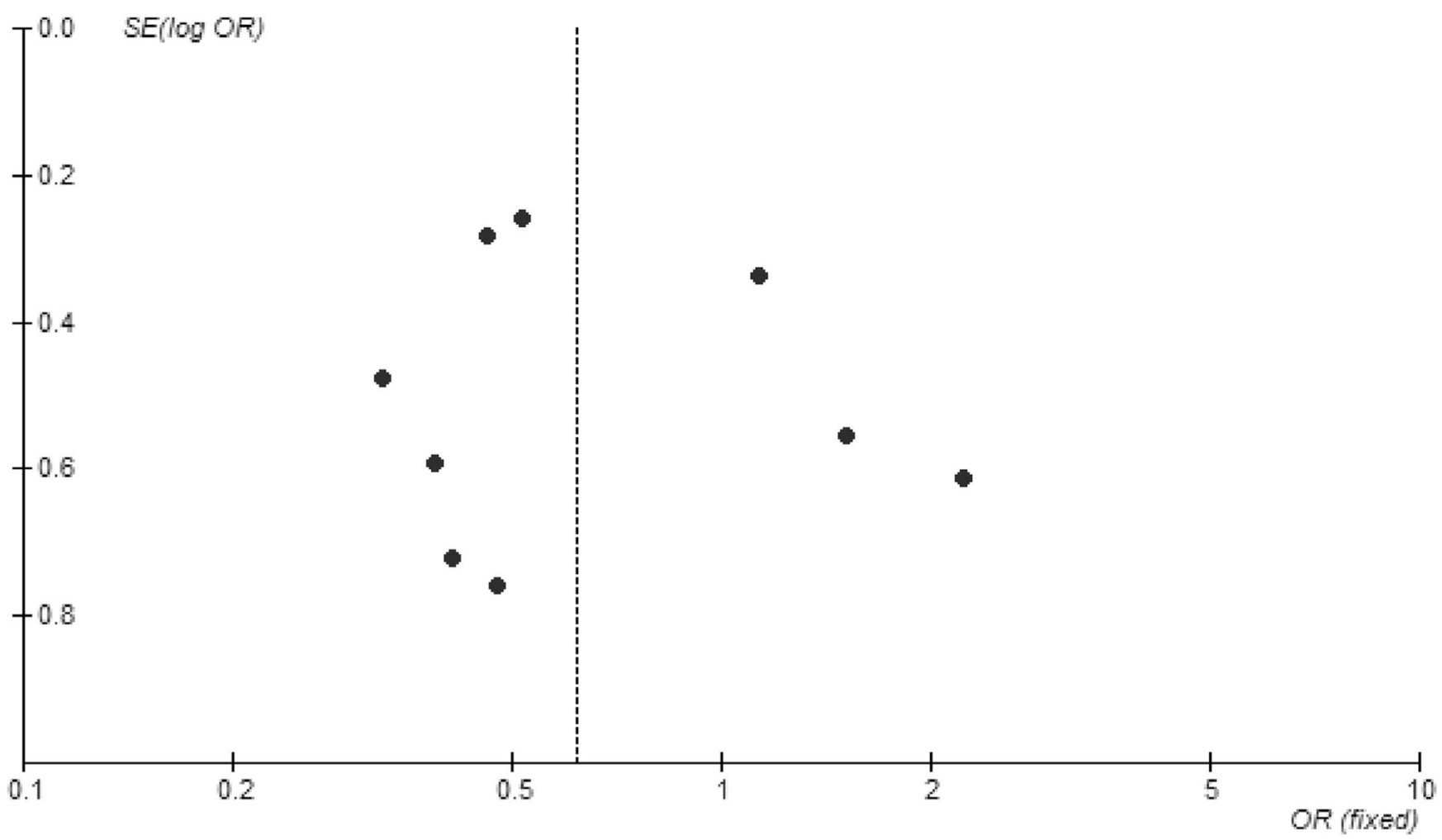

Figure 3.

Funnel plot of included studies. Individual included studies are represented by dots and are plotted by the standard error $(S E)$ of the $\log$ odds ratio $(O R)$ on the $y$-axis and the OR on the $x$-axis. The pooled OR calculated using a random effects model is represented by the vertical dotted line. This plot is provided for visual inspection for detection of publication bias. 
Primary reasons for study exclusion

Table 1

\begin{tabular}{|c|c|}
\hline & No. Reports \\
\hline \multicolumn{2}{|l|}{ After relevance screen } \\
\hline Review/letter/editorial & 220 \\
\hline Nonrandomized/nontreatment study & 450 \\
\hline Wrong focus/wrong population & 204 \\
\hline Total & 874 \\
\hline \multicolumn{2}{|l|}{ After full manuscript review } \\
\hline Review paper & 1 \\
\hline Nonrandomized & 5 \\
\hline Wrong outcome & 4 \\
\hline Total & 10 \\
\hline \multicolumn{2}{|l|}{ After author contact } \\
\hline No true experimental design & 5 \\
\hline Wrong outcome & 1 \\
\hline Wrong population & 1 \\
\hline Failed author contact & 1 \\
\hline Positive author contact but unusable data & 2 \\
\hline Total & 10 \\
\hline
\end{tabular}


Jones et al.

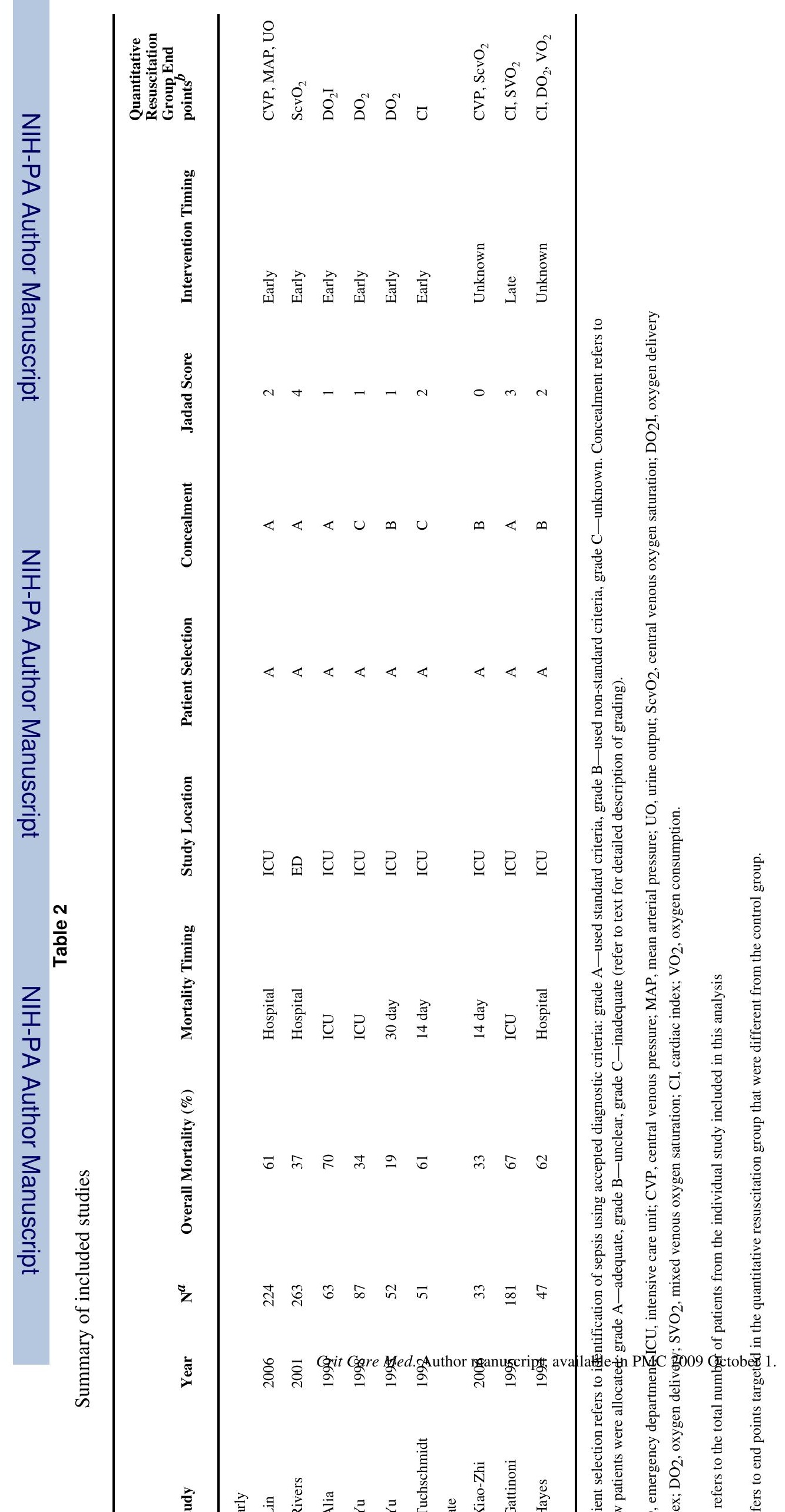

